\title{
VON HAUSARZT ZU HAUSARZT
}

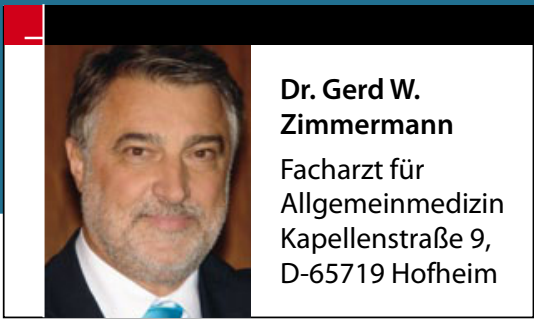

\section{Werden die AKR noch verhindert?}

- Die neuen Ambulanten Kodierrichtlinien (AKR) sollen erst zum 1.1.2012 scharf geschaltet und dann auch nur in ausgesuchten Praxen umgesetzt werden. Das hat die Vertreterversammlung der KBV am 8. April 2011 beschlossen. Der Antrag sieht vor, dass die Einführung der AKR ab dem 1.1.2012 auf einen repräsentativen Querschnitt von Arztpraxen begrenzt werden soll. Diese Praxen wiederum sollen für den nachweislich hohen Aufwand beim Kodieren einen Honorarzuschlag erhalten. Die KBV teilte mit, dass sie sich bei den Kassen dafür einsetzen will, dass diese Verschiebung der Scharfschaltung keine Konsequenzen für die Veränderungsrate der morbiditätsbedingten Gesamtvergütung im Jahr 2014 haben soll. Auch will man sich weiter bemühen, die AKR zu vereinfachen und praxistauglicher zu machen.

\section{MMW Kommentar}

Der Beschluss kommt insbesondere denjenigen Mitgliedern von Kassenärztlichen Vereinigungen zugute, die sich bisher gegen die Einführung dieses Bürokratiemonsters gewehrt und nicht in vorauseilendem $\mathrm{Ge}$ horsam kodiert haben. Umgekehrt müssen sich die Ärztinnen und Ärzte, die von ihren Vorständen zum Kodieren angehalten wurden, veräppelt fühlen. Angeblich soll ihre Arbeit irgendwann mit einem höheren Honorar belohnt werden. Tatsächlich haben sie ihre wertvolle Freizeit in ein Kodiersystem investiert, das so, wie bisher konzipiert, nicht kommen und voraussichtlich den versprochenen Honorarsegen nicht bringen wird.

\section{Extras beim Dienstwagen gelten nicht bei 1\%-Regelung}

- Der Bundesfinanzhof (BFH) hat mit Urteil vom 13.10.2010 entschieden, dass Kosten für den nachträglichen Einbau von „Extras" in ein auch zur Privatnutzung überlassenes Firmenfahrzeug nicht als Kosten für Sonderausstattung in die Bemessungsgrundlage für die sogenannte $1 \%$-Regelung einzubeziehen sind.

Im entschiedenen Fall stellte ein Unternehmen, das Flüssiggas vertreibt, seinen Außendienstmitarbeitern Firmenfahrzeuge zur Verfügung, die auch privat genutzt werden konnten. Die Umrüstkosten auf Flüssiggasbetrieb wurden nicht in die Bemessungsgrundlage der 1\%-Regelung für die private Pkw-Nutzung eingerechnet. Entsprechend wurde auch keine Lohnsteuer abgeführt. Das Finanzamt war dagegen der Auffassung, dass die Umrüstkosten in die Berechnung des geldwerten Vorteils einzubeziehen sind.

\section{MMW Kommentar}

- Der BFH bestätigte die Auffassung des Unternehmens. Die Firmenfahrzeuge waren zum Zeitpunkt der Erstzulassung nicht werkseitig mit einer Flüssiggasanlage ausgestattet. Die Kosten für den nachträglichen Einbau der Anlage sind daher nicht als Sonderausstattung in die Bemessungsgrundlage der 1\%-Regelung einzubeziehen.

Die Bemessungsgrundlage der 1\%-Regelung ist stets bezogen auf den Zeitpunkt der Erstzulassung nach dem inländischen Listenpreis zuzüglich der Kosten für die damaligen Sonderausstattungen einschließlich der Umsatzsteuer zu ermitteln. Das Urteil hat damit auch eine Auswirkung auf andere, nachträglich eingebaute Extras wie z.B. Navigationsgeräte oder auch Sondersitze.

\section{Ärztezuwanderung soll erleichtert werden}

\footnotetext{
- Ausländische Studienabschlüsse und Berufsausbildungen sollen in Deutschland künftig einfacher anerkannt werden. Das sieht das sog. Anerkennungsgesetz vor, dessen Entwurf das Bundeskabinett kürzlich verabschiedet hat. Zuwanderern, die im Ausland einen Beruf erlernt haben, wird es damit erheblich erleichtert, in Deutschland eine ihrer Qualifikation entsprechende Beschäftigung auszuüben. Mit dem Anerkennungsgesetz will die Bundesregierung dem Fachkräftemangel, der sich in vielen Bereichen abzeichnet, entgegentreten.
}

\section{MMW Kommentar}

Die Staatsangehörigkeit der Antragsteller soll bei der Bewertung der Abschlüsse künftig keine Rolle mehr spielen. Dies wird sich insbesondere auf diejenigen Berufe auswirken, bei denen die Zulassung - wie dies bei Ärzten der Fall ist - an die deutsche Staatsangehörigkeit oder die eines EU-Landes geknüpft ist. In Zukunft soll nur noch die berufliche Qualifikation ausschlaggebend sein, die der Zuwanderer mitbringt. 